\title{
224-Gb/s Carrier-recovery-free Doubly Differential 2ASK-8PSK for Short-reach Optical Networks
}

\author{
T. Zhang, C. Sanchez, M. Al-Khateeb, A. Ali, M. Tan, P. Skvortcov, I. Phillips, S. Sygletos, A. Ellis
}

\begin{abstract}
We propose and experimentally demonstrate a carrier-recovery-free 224-Gb/s dual-polarization doubly differential (DD) two-amplitude/eight-phase shift keyed (2ASK8PSK) signal for $100-\mathrm{km}$ fiber transmission with coherent detection. An 11-tap multi-symbol DD (MSDD) decoding scheme helps reduce the penalty caused by two differential operations in conventional DD decoding, allowing an optical signal-to-noiseratio (OSNR) improvement of $3.7 \mathrm{~dB}$ for DD QPSK and $4.3 \mathrm{~dB}$ for DD 16QAM. By employing such decoding, a frequency offset (FO) tolerance of $16 \mathrm{GHz}$ has been achieved in a DD 2ASK-8PSK system for a BER of $\sim 1 \times 10^{-3}$. Compared with a 224-Gb/s 16QAM system employing conventional carrier recovery algorithms, the proposed system is more robust to FOs, and the FO tolerance range is only limited by the effective receiver bandwidth.
\end{abstract}

Index Terms-Phase modulation, optical fiber communication.

\section{INTRODUCTION}

$\mathrm{T}$ HE spread of new applications such as cloud computing, augmented/virtual reality, and Internet of things has driven the demand for high-capacity short-reach networks such as metro, access and data center interconnection (DCI). For system reaches of a few hundreds of kilometers, cost and power consumption are critical for such systems design. Direct detection systems using direct modulation and advanced modulation formats $[1,2]$ have been widely investigated to realize data rates of $100 \mathrm{~Gb} / \mathrm{s}$ and beyond. To combat dispersion limits, external modulation with Kramers-Kronig (KK) receivers has been reported for larger-bandwidth DCI [3, 4]. These solutions employ a powerful digital signal processing (DSP) algorithm (i.e. KK scheme) to effectively suppress signal-signal beating interference, which requires either a high sampling rate ( $\geq 3$ Samples/symbol) [5] or iterative processing [6] to ensure acceptable system performance. Digital coherent solutions [7-9] fundamentally achieve higher capacity due to the ready access to all four field dimensions and improved signal-to-noise ratio. However, in coherent systems, the tolerated frequency offset (FO) range depends on the DSP complexity, and is commonly limited to \pm BaudRate/2M unless an unwrap function is used [10].

In [11], we reported a 200-Gb/s doubly differential (DD) encoded quadrature phase shift keying (QPSK) system for 80-

The paper was submitted in March 2018 for review. This work was partially supported by the China Scholarship Council (CSC), EU project INVENTION (659950), and EPSRC projects PEACE (EP/L000091/1), UPON (EP/M005283/1), SPFS (EP/L00044X/1). The data underlying this publication can be found at http://researchdata.aston.ac.uk/335/.

T. Zhang (zhangt16@aston.ac.uk), C. Sanchez (c.sanchezcosta@aston.ac.uk), M. Al-Khateeb (alkhamaz@aston.ac.uk), A. Ali $\mathrm{km}$ single-mode fiber transmission with simplified receiverside DSP. The system performance was practically constant for FOs within $\pm 2 \mathrm{GHz}$, which were limited by the receiver-side filter bandwidth. However, the spectral density was low due to the use of QPSK and guard bands, and the two consecutive differential operations in DD decoding also resulted in a 7.1-dB penalty, compared with a conventional QPSK system. To combat these disadvantages, we extended the principle of DD encoding on a higher-order modulation format i.e. twoamplitude/eight-phase shift keyed (2ASK-8PSK), and utilized a multi-symbol DD (MSDD) decoding scheme to effectively improve the performance of the $224-\mathrm{Gb} / \mathrm{s}$ single carrier backto-back transmission system [12].

In this paper, we provide further details of the results presented in [12], including discussion of the underlying principle of DD encoding, numerical analysis of the system performance for different modulation formats with/without DD encoding, experimental demonstration of the large FO tolerance offered by DD encoded systems as well as experimental investigation of $100-\mathrm{km}$ fiber transmission without carrier recovery. Numerical results demonstrate that 11-tap MSDD decoding allows optical signal-to-noise ratio (OSNR) improvement of $3.7 \mathrm{~dB}$ in DD QPSK and $4.3 \mathrm{~dB}$ in DD 16 quadrature amplitude modulation (16QAM) systems. It also enables DD 2ASK-8PSK to have a 3.4-dB receiver sensitivity advantage over DD 16QAM. Experimental results demonstrate good robustness to FO with the tolerance of $16 \mathrm{GHz}$ for the 28Gbaud/s DD encoding systems at a bit-error-rate (BER) of $1 \times 10^{-3}$, which is twice that of QPSK and 16QAM systems with conventional carrier recovery. Offset against these advantages, we find that due to constellation design and differential operations in the DD decoding, the required OSNR for 224$\mathrm{Gb} / \mathrm{s}$ DD 2ASK-8PSK at a BER of $1 \times 10^{-3}$ is $5.2 \mathrm{~dB}$ higher than that of a 16QAM system.

\section{PRINCIPLE FOR DD 2ASK-8PSK ENCODING}

Carrier synchronization is commonly required in coherent systems, but it can be readily eliminated by employing DD decoding. Only two consecutive one-symbol delay integration and differentiation operations are required in the precoding and

(aliaai@aston.ac.uk), M. Tan (m.tan1@aston.ac.uk), P. Skvortcov (skvortcp@aston.ac.uk), I. Phillips (i.phillips@aston.ac.uk), S. Sygletos (s.sygletos@aston.ac.uk) and A. D. Ellis (andrew.ellis@aston.ac.uk) are with Aston Institute of Photonic Technologies, Aston University, Birmingham, B4 7ET, UK. 


\section{(a)}

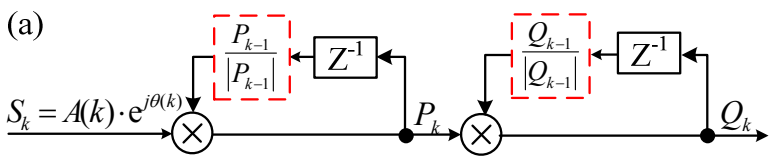

(b)

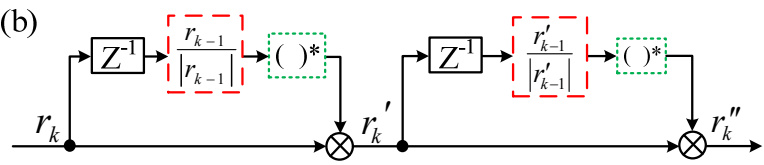

Fig. 1. (a) Precoding and (b) conventional decoding for doubly differential encoded higher-order modulation formats. The normalization and conjugation operations are represented as red dashed and green dotted blocks respectively.

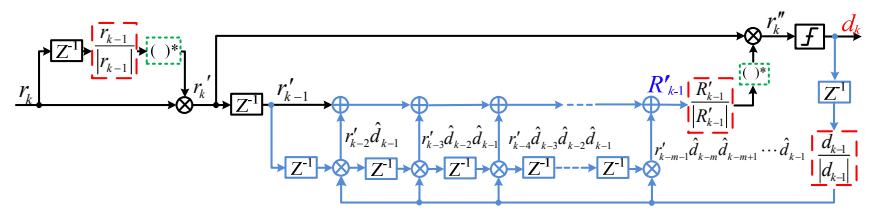

Fig. 2. MSDD decoding scheme with multi-symbol decision feedback.

conventional decoding of a DD QPSK signal [11]. To implement doubly differential encoding on higher-order modulation formats with multiple radii, normalization operations (red dashed_blocks in Fig. 1(a)) are required in each differential precoding stage in order to pre-code only the phase information. The generated doubly differential encoded signal $\left(Q_{k}\right)$ can be written as:

$Q_{k}=A(k) \cdot e^{j \varphi_{D D E}(k)}$

$$
=A(k) \cdot e^{j\left(\theta(k)+2 * \varphi_{D D E}(k-1)-\varphi_{D D E}(k-2)\right)}
$$

Here, $k$ refers to the symbol index; $A$ and $\theta$ are the amplitude and phase of the original symbol $(S)$ respectively; $\varphi_{D D E}$ is the DD pre-coded symbol's phase. Equation (1) shows that the original amplitude information is retained whilst the phase information is integrated twice.

Conventionally, two consecutive normalized differential demodulation operations can be employed in the receiver to recover the original signal and simultaneously remove fixed frequency offset and phase noise. Specifically, the received signal $\left(r_{k}\right)$ after coherent detection has an unknown frequency offset $(\omega)$ and phase noise $\left(\emptyset_{n}(k)\right)$, and can be written as:

$r_{k}=A(k) \cdot \mathrm{e}^{j\left(\varphi_{D D E}(k)+k \omega T+\emptyset_{n}(k)\right)}$

where $T$ refers to the symbol duration. Assuming slowly varying phase noise, the signal after the $1^{\text {st }}$ normalized differential operation becomes:

$r_{k}^{\prime}=A(k) \cdot \mathrm{e}^{j\left(\varphi_{D D E}(k)-\varphi_{D D E}(k-1)+\omega T\right)}$

From (3), we can see that the $1^{\text {st }}$ normalized differential operation mitigates the impact of phase noise and converts the FO into a phase offset, which can be eliminated in the $2^{\text {nd }}$ normalized differential stage. The final signal can be written as: $r_{k}^{\prime \prime}=A(k) \cdot \mathrm{e}^{j\left(\varphi_{D D E}(k)+\varphi_{D D E}(k-2)-2 * \varphi_{D D E}(k-1)\right)}$

which is expected to be the same as the original signal $\left(S_{k}\right)$. However, this conventional decoding scheme results in a large receiver sensitivity penalty (e.g. 7.17-dB penalty when employing DD encoding on QPSK [13]) due to aggravated noise power from the two differential operations. In this paper, we employed MSDD decoding with multi-symbol decision feedback [14] included in the $2^{\text {nd }}$ differential stage to mitigate this degradation. Specifically, instead of using only one received noisy symbol (e.g. $r_{k-1}^{\prime}$ in Fig. 1(b)) as phase reference for the symbol of interest, an improved reference signal is generated for the $2^{\text {nd }}$ differential operation:

$R_{k-1}^{\prime}=r_{k-1}^{\prime}+r_{k-2}^{\prime} \hat{d}_{k-1}+r_{k-3}^{\prime} \hat{d}_{k-2} \hat{d}_{k-1}+$

$r_{k-4}^{\prime} \hat{d}_{k-3} \hat{d}_{k-2} \hat{d}_{k-1}+\cdots+r_{k-m-1}^{\prime} \hat{d}_{k-m} \hat{d}_{k-m+1} \cdots \hat{d}_{k-1}$

, using several additional consecutive symbols in combination with the decided symbols' phase $\left(\hat{d}_{k-i}, i=1,2, \ldots m\right)$, as depicted in Fig. 2. Here, $m$ is the memory length of this feedback process. In (5), each term is roughly aligned in-phase with $r_{k-1}^{\prime}$, thus the average of the summation mitigates noise and gives a better symbol reference $\left(R_{k-1}^{\prime}\right)$. The complex multipliers for these references generation can be realized by lookup tables [14], leading to reduced DSP complexity.

\section{RESULTS AND DISCUSSION}

\section{A. Simulation results}

We firstly numerically investigated the performance of 28Gbaud/s signals with different modulation formats (QPSK, DD QPSK, 16QAM, DD 16QAM, and the proposed DD 2ASK8PSK with conventional DD or MSDD decoding) over a white Gaussian noise channel at zero frequency offset. The 28Gbaud/s QPSK, 16QAM or 2ASK-8PSK signals (normalized radii of 0.74 and 1.205 with zero phase offset between 8PSK signals on two rings) were firstly Gray mapped from $2^{15}-1$ pseudo random binary sequences. Then DD precoding, as shown in Fig. 1(a), was symbol-wise implemented in the polar coordinate. After transmission over an additive white Gaussian
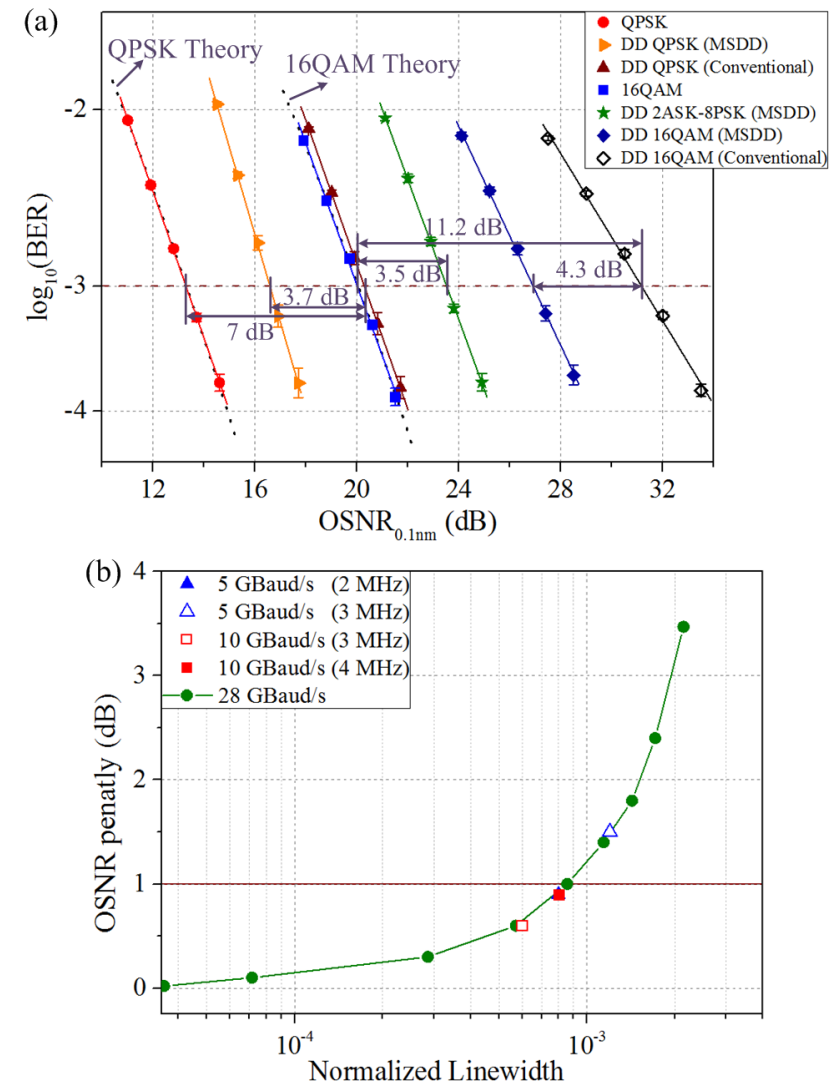

Fig. 3. (a) OSNR performance for $28 \mathrm{Gbaud} / \mathrm{s}$ system employing QPSK, DDQPSK, 16QAM, DD16QAM or DD 2ASK-8PSK with conventional DD or MSDD $(\mathrm{m}=10)$ decoding at zero FO. (b) Linewidth tolerance for DD 2ASK8PSK with 11-tap MSDD decoding at zero FO, different symbol rates (outside brackets in legend) and different laser linewidths (inside legend brackets). 
noise channel, the DD pre-coded signals were recovered using either conventional DD $(m=0)$ or MSDD $(m \geq 1)$ decoding. The system performance was evaluated by calculating the average BER and its standard deviation from 655,360 symbols for each OSNR (measured at 0.1-nm resolution bandwidth).

Figure 3(a) shows that for both QPSK and 16QAM, the simulated OSNR performance matches very well with theoretical predictions (black dotted lines). With conventional DD decoding, the DD QPSK system suffered from an OSNR penalty of $7 \mathrm{~dB}$ at BER of $1 \times 10^{-3}$, which is close to the theoretical expectation $(7.17 \mathrm{~dB})$, whilst the DD 16QAM system was degraded by $11.2 \mathrm{~dB}$, in comparison to the systems without DD encoding. MSDD decoding with a decision feedback length of ten in the $2^{\text {nd }}$ differential stage effectively reduced the OSNR penalty in DD QPSK and DD 16QAM systems by $3.7 \mathrm{~dB}$ and $4.3 \mathrm{~dB}$ respectively. Nevertheless, large performance degradation of $6.9 \mathrm{~dB}$ still remains in DD 16QAM system, due to poor phase error tolerance for the eight points on the $2^{\text {nd }}$ ring of 16QAM. By contrast, the constellation points of the 2ASK-8PSK signal are more uniformly distributed in phase, giving better phase noise tolerance. With the same MSDD decoding, DD 2ASK-8PSK system only suffers a 3.5-dB penalty when compared to 16QAM, which is slightly larger than the penalty between differential QPSK and QPSK. Utilizing the same numerical method as that in [11], a large linewidth tolerance of $\sim 8.6 \times 10^{-4}$ (each laser linewidth of $\sim 12$ MHz for 28 Gbaud) at 1-dB OSNR penalty was achieved in Fig. 3 (b), indicating that low-cost commercial lasers with several MHz linewidth can be used in the 28-Gbaud/s DD 2ASK-8PSK transceiver without significant OSNR performance degradation.

\section{B. Experimental verification}

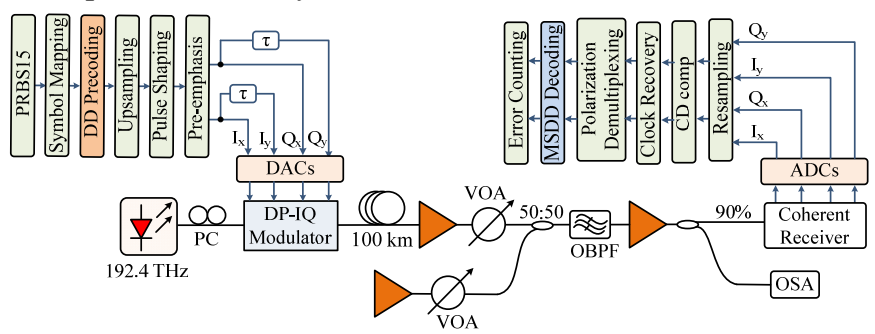

Fig. 4. Experimental setup for 224-Gb/s dual-polarization (DP) DD 2ASK8PSK signal transmission over 100-km single-mode fiber with 11-tap MSDD decoding used in the receiver. EDFA: erbium-doped fiber amplifier; VOA: variable optical attenuators; OBPF: optical bandpass filter.

We also verified the feasibility of the $224-\mathrm{Gb} / \mathrm{s}$ dualpolarization DD 2ASK-8PSK signal for back-to-back (B2B) and $100-\mathrm{km}$ fiber transmission with 11-tap MSDD $(\mathrm{m}=10)$ decoding in the receiver, using the experimental setup shown in Fig. 4. The measurement of DD 16QAM signal was excluded due to the high required OSNR. The $28-$ Gbaud/s DD pre-coded 2ASK-8PSK signal was up-sampled to 2 Samples/symbol and pulse shaped using a square root raised cosine filter with a rolloff factor of 0.3. Digital pre-emphasis for the arbitrary waveform generator's frequency response was performed utilizing the manufacturer's calibration files. An inverse transfer function of the IQ modulator was implemented to reduce the impact caused by the nonlinearity of the driver amplifiers. The $\mathrm{Y}$ polarization signal was de-correlated by delaying the X polarization signal by 8192 samples, both of which were loaded to the memories of four digital to analogue converters (DACs) running at $56 \mathrm{GS} / \mathrm{s}$. The OSNR was measured using a high-resolution $(150 \mathrm{MHz})$ optical spectrum analyzer (OSA) and the spectral integration method [15] with a measurement bandwidth of $80 \mathrm{GHz}$ and a linear curve fitted noise floor for noise power calculation. Due to slight nonlinearity in the OSA power measurement, a linearity correction factor (0.978) was applied to all measured OSNRs.

A coherent receiver (Tektronix, OM4245) with a freerunning local oscillator was used to detect the signal. Offline DSP for the data captured at $100 \mathrm{GS} / \mathrm{s}$ included resampling to 2 Samples/symbol, chromatic dispersion compensation, clock recovery and polarization de-multiplexing, which was implemented using eight 25-tap adaptive finite impulse response filters with real-valued weights optimized through a radius-directed equalizer. Instead of implementing carrier recovery, which was required in QPSK and 16QAM systems, MSDD decoding with decision feedback length of 10 was employed in DD 2ASK-8PSK system. The average BER and its standard deviation were obtained by independent processing on ten traces $(\sim 100,000$ symbols for each trace $)$.

Figure 5 compares the frequency offset tolerance of different modulation formats by changing the frequency of the local oscillator (LO). For 28-Gbaud/s QPSK and 16QAM, the DD pre-coder was omitted, and the MSDD decoding in the proposed receiver was replaced by a fast Fourier transform based FO compensator [16] as well as a one-filter (averaging window size of 61) based decision-directed soft-decision phase estimator [17]. As depicted in Fig. 5, a limited FO range of $\sim 7$ $\mathrm{GHz}( \pm 3.5 \mathrm{GHz})$ is observed in QPSK and 16QAM systems, in line with theoretical expectation $( \pm 28 / 2 / 4)$ due to the fourth power operation. At the edge of the FO tolerance range, both system performance become unstable due to the wavelength drifting of the LO, leading to large standard deviation of BER (not shown in Fig. 5). In contrast, DD encoding allows an increased FO tolerance range of $16 \mathrm{GHz}$ at a BER of $\sim 1 \times 10^{-3}$, which is limited only by the effective bandwidth of the receiver. To achieve this FO tolerance in conventional coherent systems, nonlinear unwrap function [10] would be required.

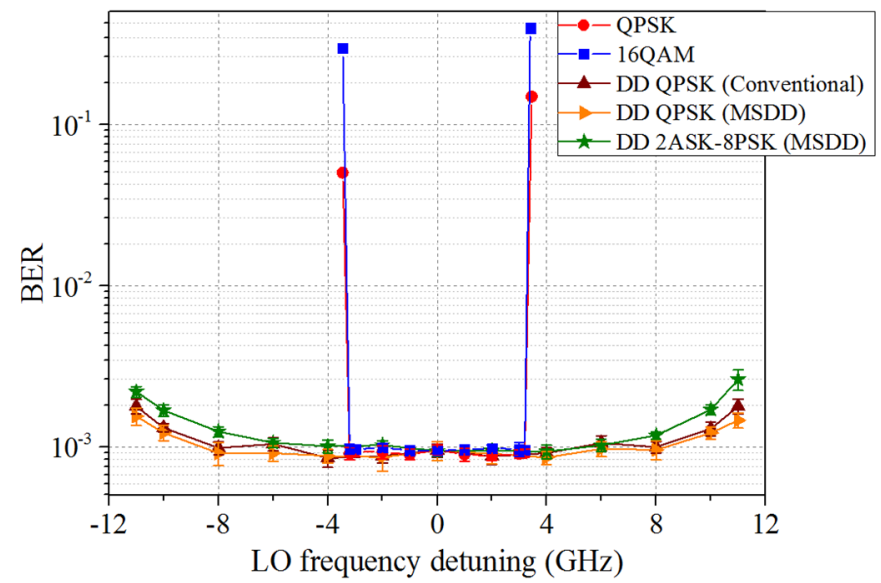

Fig. 5. Frequency offset tolerance of 28-Gbaud/s B2B systems using QPSK, 16QAM (both with full carrier recovery), DD QPSK with conventional DD or MSDD decoding, and DD 2ASK-8PSK with MSDD decoding at measured OSNR of $13.9 \mathrm{~dB}, 21.9 \mathrm{~dB}, 21.8 \mathrm{~dB}, 17.5 \mathrm{~dB}$, and $27.1 \mathrm{~dB}$ respectively. 

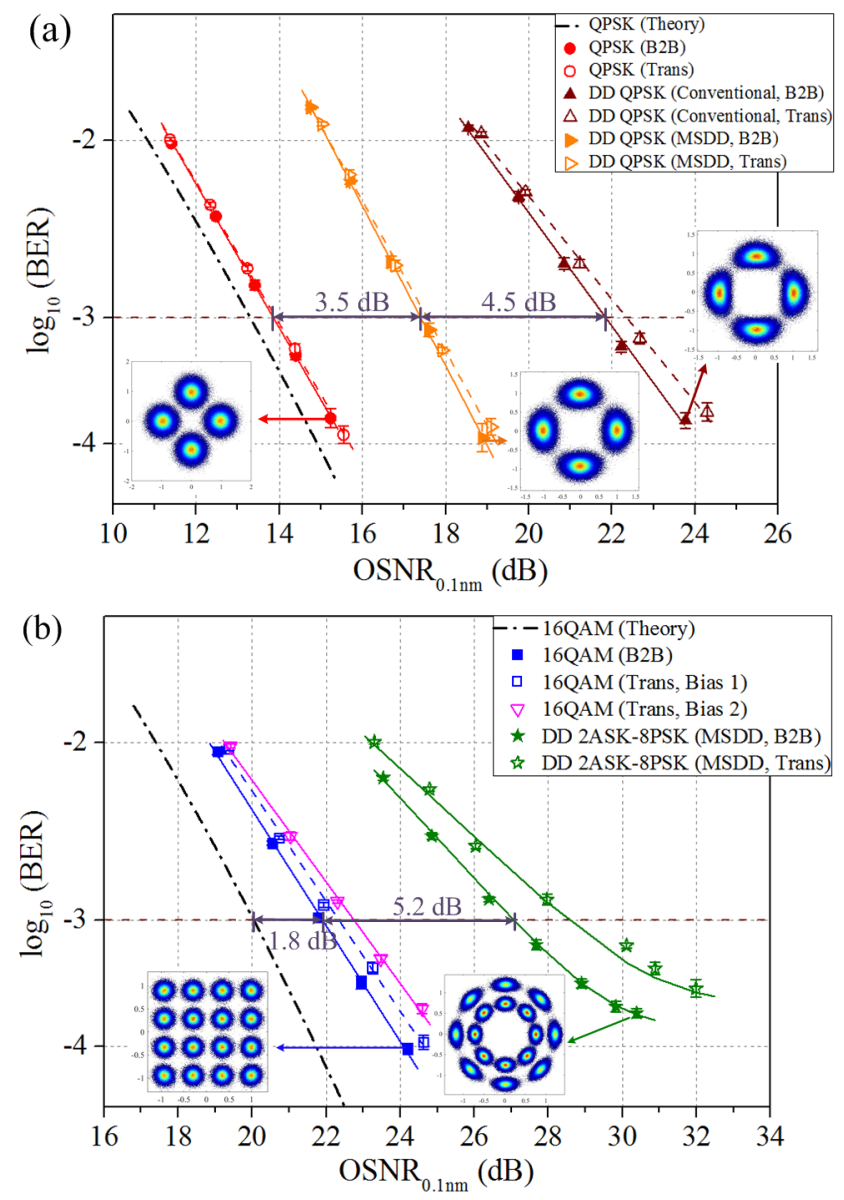

Fig. 6. B2B and 100-km fiber transmission performance of dual-polarization 28-Gbaud/s systems using (a) QPSK or DD QPSK with conventional DD or MSDD $(\mathrm{m}=10)$ decoding, and (b) 16QAM or DD 2ASK-8PSK with MSDD $(\mathrm{m}=10)$ decoding.

We also investigated the transmission performance of the different modulation formats. As shown in Fig. 6(a), the implementation penalty for the 224-Gb/s QPSK system is 0.6 $\mathrm{dB}$, and conventional $\mathrm{DD}$ decoding resulted in an 8-dB (theoretically 7.17-dB) OSNR penalty at BER of $1 \times 10^{-3}$. By resorting to MSDD decoding with 10 -symbol decision feedback, the OSNR performance was only $3.5 \mathrm{~dB}$ away from QPSK system with carrier recovery, which is only $\sim 1 \mathrm{~dB}$ worse than differential QPSK. For QPSK signal with/without DD encoding after $100-\mathrm{km}$ fiber transmission, no severe performance degradation is observed in Fig. 6(a). Figure 6(b) shows an implementation penalty of $1.8 \mathrm{~dB}$ for $28-\mathrm{Gbaud} / \mathrm{s}$ 16QAM, and a 5.2-dB required OSNR difference between 16QAM with carrier recovery and DD 2ASK-8PSK with 11-tap MSDD decoding. Without MSDD decoding, DD 2ASK-8PSK was not detectable at the OSNR levels reported here. For 100$\mathrm{km}$ fiber transmission, the transmission penalty for 16QAM system (blue hollow square) is negligible, whilst the DD 2ASK8PSK signal suffered from a $1.5-\mathrm{dB}$ OSNR penalty at a BER of $1 \times 10^{-3}$. As shown in Fig. 6(b), the transmission penalty for 16QAM with carrier recovery depends on the accuracy of the bias point. We believe that this coupled with the accuracy of modulator nonlinearity compensation are responsible for the higher transmission penalty for DD 2ASK-8PSK.

\section{CONCLUSION}

We have experimentally demonstrated a $224-\mathrm{Gb} / \mathrm{s}$ carrierrecovery-free coherent system using DD 2ASK-8PSK. The conventional DD decoding induced OSNR penalty can be reduced by $3.7 \mathrm{~dB}$ for DD QPSK and $4.3 \mathrm{~dB}$ for DD 16QAM using an 11-tap MSDD decoding scheme, which also allows a large FO tolerance range of $16 \mathrm{GHz}$ at the BER of $1 \times 10^{-3}$. This range is twice that of the 16QAM system with conventional carrier recovery at cost of a 5.2-dB performance degradation.

\section{REFERENCES}

[1] T. Takahara, et al., "Discrete Multi-Tone for $100 \mathrm{~Gb} / \mathrm{s}$ Optical Access Networks," Proc. OFC, San Francisco, CA, 2014, paper M2I.1.

[2] K. P. Zhong, et al., "140-Gb/s 20-km Transmission of PAM-4 Signal at 1.3 $\mu \mathrm{m}$ for Short Reach Communications," IEEE Photon. Technol. Lett., vol. 27, no. 16, pp. 1757-1760, 2015.

[3] X. Chen, et al., "218-Gb/s Single-Wavelength, Single-Polarization, SinglePhotodiode Transmission Over 125-km of Standard Singlemode Fiber Using Kramers-Kronig Detection," in Proc. OFC, Los Angeles, CA, 2017, paper Th5B.6.

[4] S. T. Le, et al., "1.72-Tb/s Virtual-Carrier-Assisted Direct-Detection Transmission Over 200 km," J. Lightwave Technol., vol. 36, no. 6, pp. 1347-1353, 2018.

[5] Z. Li, et al., "Joint optimization of resampling rate and carrier-to-signal power ratio in direct-detection Kramers-Kronig receivers," in Proc. ECOC, Gothenburg, Sweden, 2017, paper W.2.D.3.

[6] A. Mecozzi, et al., "Kramers-Kronig coherent receiver," Optica, vol. 3, pp. 1220-1227, 2016.

[7] H. Chien, et al., "On single-carrier 400G line side optics using PM256QAM," in Proc. ECOC, Dusseldorf, Germany, 2016, pp. 1-3.

[8] P. J. Winzer, et al., "Generation and 1,200-km transmission of 448-Gb/s ETDM 56-Gbaud PDM 16-QAM using a single I/Q modulator," in Proc. ECOC, Torino, 2010, pp. 1-3.

[9] X. Liu, et al., "448-Gb/s Reduced-Guard-Interval CO-OFDM Transmission Over $2000 \mathrm{~km}$ of Ultra-Large-Area Fiber and Five 80-GHzGrid ROADMs," J. Lightwave Technol., vol. 29, no. 4, pp. 483-490, 2011.

[10]R. M. Ferreira, et al., "Optimized Carrier Frequency and Phase Recovery Based on Blind M th Power Schemes," IEEE Photon. Technol. Lett., vol. 28, no. 21, pp. 2439-2442, 2016.

[11]T. Zhang, et al., "Amplifier-free 200-Gb/s tandem SSB doubly differential QPSK signal transmission over 80-km SSMF with simplified receiver-side DSP," Opt. Express, vol. 26, no. 7, pp. 8418-8430, 2018.

[12]T. Zhang, et al., "224Gb/s Single Carrier Doubly Differential 2ASK-8PSK System without Carrier Recovery," in Proc. CLEO, San Jose, 2018, paper SM2C.6.

[13] A. J. Walsh, et al., "Highly Robust Dual-Polarization Doubly Differential PSK Coherent Optical Packet Receiver for Energy Efficient Reconfigurable Networks," J. Lightwave Technol., vol. 33, no. 24, pp. 5218-5226, 2015.

[14]I. Tselniker, et al., "Joint phase noise and frequency offset estimation and mitigation for optically coherent QAM based on adaptive multi-symbol delay detection (MSDD)," Opt. Express, vol. 20, no. 10, pp. 10944-10962, 2012.

[15] Available at https://www.viavisolutions.com/en-us/literature/band-osnrmeasurements-40-g-polarization-multiplexed-qpsk-signals-using-fielddeploy-white-paper-en.pdf.

[16]Y. Wang, et al., "Non-Data-Aided Feedforward Carrier Frequency Offset Estimators for QAM Constellations: A Nonlinear Least-Squares Approach," EURASIP J. Adv. Sig. Pr., vol. 13, pp. 1993-2001, 2004.

[17]E. Ip, et al., "Feedforward Carrier Recovery for Coherent Optical Communications," J. Lightwave Technol., vol. 25, no. 9, pp. 2675-2692, 2007. 